\section{Robust and Minimum Norm Pole Assignment with Periodic State Feedback}

\author{
Andras Varga
}

\begin{abstract}
A computational approach is proposed to solve the minimum norm or robust pole assignment problem for linear periodic discrete-time systems. The proposed approach uses a periodic Sylvester-equation-based parametrization of the periodic pole assignment problem and exploits the nonuniqueness of the problem by imposing conditions on the norm of the resulting periodic state feedback or on the condition numbers of the periodic eigenvector matrices of the closed-loop system. The solution method relies on using gradient search methods on suitably defined cost functions. Explicit expressions of the gradients of cost functions are derived, and the efficient evaluation of the cost functions and gradients is discussed. Numerical examples illustrate the effectiveness of the proposed approach.
\end{abstract}

Index Terms-Discrete-time systems, numerical methods, periodic systems, robust pole assignment.

\section{INTRODUCTION}

Pole assignment techniques to modify the dynamic response of a linear system are among the most studied problems in modern control theory. The complete theoretical solution of this problem for standard systems has been followed by the development of many computational methods (see, for example, the collection of reprints in [13]). Sensitivity analysis of the pole assignment problem (see [10] and references therein) moves one step forward to the understanding of difficulties and practical limitations associated with the usage of solution methods.

Periodic systems represent the simplest form of the more general time-varying systems. The interest for this class of systems has been motivated by the advent of multirate sampled-data systems as well as by the study of genuine periodic systems describing cyclic physical dynamical behaviors. Most theoretical concepts and several numerical methods for standard discrete-time systems have been extended to the case of periodic systems. One such example is the pole assignment problem for periodic systems discussed theoretically in [7] and [4] and addressed as well from a computational point of view in [14] and [9].

Consider the linear discrete-time periodic system of the form

$$
x_{k+1}=A_{k} x_{k}+B_{k} u_{k}
$$

where the matrices $A_{k} \in \mathbb{R}^{n \times n}$ and $B_{k} \in \mathbb{R}^{n \times m}$ are periodic with period $K \geq 1$. We consider the following periodic eigenvalue assignment problem (PEAP): given the completely reachable periodic matrix pair $\left(A_{k}, B_{k}\right)$, determine the periodic feedback matrix $F_{k} \in$ $\mathbb{R}^{m \times n}$ such that the eigenvalues of the closed-loop monodromy matrix $\Phi_{A+B F}(K, 0)=\left(A_{K-1}+B_{K-1} F_{K-1}\right) \cdots\left(A_{0}+B_{0} F_{0}\right)$ are at desired locations $\Gamma=\left\{\lambda_{1}, \cdots, \lambda_{n}\right\}$ in the complex plane. We assume that $\Gamma$ is symmetric with respect to the real axis. This assumption guarantees that the resulting periodic matrix $F_{k}$ can be chosen real.

In the multi-input case or when $K>1$, the PEAP has a nonunique solution. Therefore, it is reasonable to exploit the nonuniqueness by imposing additional conditions. One aspect that is desirable from a practical point of view is to determine feedback matrices with small gains.

Manuscript received August 13, 1998; revised May 13, 1999. Recommended by Assoicate Editor, E. Feron.

The author is with the German Aerospace Center, DLR-Oberpfaffenhofen, Institute of Robotics and System Dynamics, D-82234 Wessling, Germany (e-mail: andras.varga@ dir.de).

Publisher Item Identifier S 0018-9286(00)00826-6.
Intuitively, this must be advantageous because small feedback gains lead to smaller control signals and, thus, to less energy consumption. Small gains are also beneficial to reduce noise amplification. A second aspect important in pole assignment is to achieve small condition numbers for the eigenvector matrices of the closed-loop system. This is the goal of robust pole assignment [5], [9]. Both of these aspects are decisive for the overall sensitivity of assigned eigenvalues, because, as was shown in the standard systems case [10], high feedback gains or high condition numbers lead to increased sensitivity of the closed-loop eigenvalues. It appears that the simultaneous minimization of the feedback norm and condition of eigenvector matrix is a desirable general goal for solving the PEAP.

In this correspondence, we address the problem of determining the minimum norm periodic state feedback, which solves the PEAP and simultaneously minimizes the sensitivity of the closed-loop eigenvalues. The feedback norm minimization in standard pole assignment problem has been considered in [6]. Here, a Sylvester-equation-based parametrization of the eigenvalue assignment problem is used, and the minimum Frobenius-norm feedback is computed by performing a gradient search on the free problem parameters. This approach has been improved in [15] by eliminating the technical constraints on the problem and by improving tremendously the computational efficiency. Note that the use of Frobenius-norm allows us to derive the explicit analytic expression of the gradient of the norm of the feedback, thus making easier the usage of gradient-based minimization techniques. Therefore, the same choice of norm will be used also for periodic systems.

We propose a computational approach to solve the PEAP that extends the method for standard systems of [15] and addresses simultaneously the minimum norm and robust pole assignment aspects. By using a periodic Sylvester-equation-based parametrization, the PEAP is formulated as an unconstrained parametric minimization problem for a suitably chosen cost function depending on a set of free parameters. The derived explicit expressions of gradients of some cost functions allow the efficient use of powerful unconstrained minimization methods based on gradient search techniques. Further, we discuss the numerical aspects of evaluating the cost functions and their gradients. Each function/gradient evaluation involves the solution of two periodic Sylvester equations. Efficient, numerically stable algorithms to solve such equations are based on the use of the periodic Schur form and described in [3]. A specialized version of the general algorithm of [3] has been derived, representing the direct generalization of the well known Bartels-Stewart method to solve standard Sylvester equations [1]. We show how to use this algorithm to make cost and gradient evaluations highly efficient. Finally, we present some numerical examples to illustrate the effectiveness of the proposed approach to solve minimum norm, robust pole assignment problems.

Notation: For a square time-varying matrix $A_{k}, k=0,1, \cdots$, we denote $\Phi_{A}(j, i)=A_{j-1} A_{j-2} \cdots A_{i}$ for $j>i$ and $\Phi_{A}(i, i):=I$. If $A_{k}$ is periodic with period $K$, then the monodromy matrix of the system (1) at time $\tau$ is $\Phi_{A}(\tau+K, \tau)$. Its eigenvalues, denoted by $\Lambda\left(\Phi_{A}(\tau+K, \tau)\right)$, are independent of $\tau$ and called characteristic multipliers. For an arbitrary periodic matrix $X_{k}$ of period $K$, also called a $K$-periodic matrix, we use alternatively the script notation $\mathcal{X}$, which associates the block-diagonal matrix $\mathcal{X}=\operatorname{diag}\left(X_{0}, X_{1}, \cdots, X_{K-1}\right)$ with the cyclic sequence of matrices $X_{k}, k=0, \cdots, K-1$. This notation is consistent with the standard matrix operations like addition, multiplication, transposing, or inversion. Further, we denote with $\sigma \mathcal{X}$ the $K$-cyclic shift $\sigma \mathcal{X}=\operatorname{diag}\left(X_{1}, \cdots, X_{K-1}, X_{0}\right)$ applied to the cyclic sequence $X_{k}, k=0, \cdots, K-1$. The notation $\mathcal{X}_{i j}$ is used to refer simultaneously to all $(i, j)$ elements or to all $(i, j)$ blocks of the 
cyclic sequence $X_{k}, k=0, \cdots, K-1$. With a little abuse, we will also use this notation to denote the submatrices of partitioned periodic matrices.

\section{COMPUTATION OF RoBUST AND MINIMUM NORM FEEDBACK}

To solve the PEAP, we use a straightforward parametrization of the pole assignment problem. Let $G_{k} \in \mathbb{R}^{m \times n}$ be a given $K$-periodic parameter matrix, and let $\tilde{A}_{k} \in \mathbb{R}^{n \times n}$ be a given $K$-periodic matrix such that $\Lambda\left(\Phi_{\tilde{A}}(K, 0)\right)=\Gamma$, where $\Gamma$ is the desired set of closed-loop characteristic values. Consider the following periodic Sylvester equation (PSE)

$$
A_{k} X_{k}-X_{k+1} \tilde{A}_{k}+B_{k} G_{k}=0, \quad k=0, \cdots, K-1
$$

and assume that its solution, the $K$-periodic matrix $X_{k}$, is invertible. Then, with the periodic feedback matrix

$$
F_{k}=G_{k} X_{k}^{-1}
$$

we have $X_{0}^{-1} \Phi_{A+B F}(K, 0) X_{0}=\Phi_{\tilde{A}}(K, 0)$, and thus, $F_{k}$ solves the PEAP. Usual restrictions on choosing $\tilde{A}_{k}$ and $G_{k}$ are similar to those in the standard case [6]: 1) the periodic pair $\left(\tilde{A}_{k}, G_{k}\right)$ is completely observable; and 2) $\Lambda\left(\Phi_{A}(K, 0)\right) \cap \Lambda\left(\Phi_{\tilde{A}}(K, 0)\right)=\emptyset$. If, additionally, the periodic pair $\left(A_{k}, B_{k}\right)$ is completely reachable, then $X_{k}$ satisfying 2) is generically nonsingular, and for the above $F_{k}$, we have $X_{k+1}^{-1}\left(A_{k}+B_{k} F_{k}\right) X_{k}=\tilde{A}_{k}$. To solve the robust pole assignment problem, we also assume that the resulting closed-loop monodromy matrix $\Phi_{A+B F}(K, 0)$ is nondefective. This result can be achieved by choosing, for instance, $\tilde{A}_{0}$ in a real Jordan form with only $1 \times 1$ or $2 \times 2$ diagonal blocks and $\tilde{A}_{k}, k=1, \cdots, K-1$, diagonal. In this case, each $X_{k}$ is also an eigenvector matrix for the corresponding monodromy matrix $\Phi_{A+B F}(k+K, k)$. Although redundant, the above parametrization has the main advantage to allow the derivation of analytic expressions of gradients of many useful cost functions that can be formulated to eliminate the intrinsic nonuniqueness of the PEAP.

To solve the minimum norm, robust PEAP consider the following cost function to be minimized:

$$
J=\alpha \frac{1}{2} \sum_{k=0}^{K-1} \kappa_{F}^{2}\left(X_{k}\right)+(1-\alpha) \frac{1}{2} \sum_{k=0}^{K-1}\left\|F_{k}\right\|_{F}^{2}
$$

where $\kappa_{F}\left(X_{k}\right):=\left\|X_{k}\right\|_{F} \cdot\left\|X_{k}^{-1}\right\|_{F}$ is the Frobenius-norm condition number of $X_{k}$ and $0 \leq \alpha \leq 1$ is a weighting factor. Notice that with $\alpha=0, J$ defines a norm minimization problem, and with $\alpha=1$, we get a pure robust pole assignment problem. Intermediary values of $\alpha$ lead to a combination of both aspects.

$J$ can be expressed alternatively as

$$
\begin{aligned}
J= & \alpha \frac{1}{2} \sum_{k=0}^{K-1} \operatorname{tr}\left[X_{k}^{T} X_{k}\right] \operatorname{tr}\left[X_{k}^{-T} X_{k}^{-1}\right] \\
& +(1-\alpha) \frac{1}{2} \sum_{k=0}^{K-1} \operatorname{tr}\left[F_{k}^{T} F_{k}\right]
\end{aligned}
$$

or, by using the script notation, as

$$
\begin{aligned}
J= & \alpha \frac{1}{2} \sum_{k=0}^{K-1} \operatorname{tr}\left[\mathcal{I}_{k} \mathcal{X}^{T} \mathcal{X}\right] \operatorname{tr}\left[\mathcal{I}_{k} \mathcal{X}^{-T} \mathcal{X}^{-1}\right] \\
& +(1-\alpha) \frac{1}{2} \operatorname{tr}\left[\mathcal{F}^{T} \mathcal{F}\right]
\end{aligned}
$$

where $\mathcal{I}_{k}$ is a block diagonal matrix with all diagonal blocks equal to zero with the exception of $k$ th block, which is equal to $I_{n}$. Using the proposed parametrization, $\mathcal{F}$ is computed as

$$
\mathcal{F}=\mathcal{G} \mathcal{X}^{-1}
$$

where $\mathcal{X}$ satisfies the PSE

$$
\mathcal{A X}-\sigma \mathcal{X} \tilde{\mathcal{A}}+\mathcal{B \mathcal { G }}=0
$$

The gradient of $J$ with respect to $\mathcal{G}$ can be computed by employing the following result.

Proposition 1: Let $\mathcal{F}$ be the periodic feedback computed as in (5), assigning the desired characteristic values $\Gamma$ for given $\tilde{\mathcal{A}}$ and $\mathcal{G}$. Then, the gradient of $J$ with respect to $\mathcal{G}$ is given by

$$
\begin{aligned}
\nabla_{\mathcal{G}} J= & (1-\alpha)\left(\mathcal{H}^{T}-\mathcal{B}^{T} \mathcal{U}^{T}\right)+\alpha \sum_{k=0}^{K-1} \operatorname{tr}\left[X_{k}^{-T} X_{k}^{-1}\right] \mathcal{B}^{T} \mathcal{V}_{k}^{T} \\
& +\alpha \sum_{k=0}^{K-1} \operatorname{tr}\left[X_{k}^{T} X_{k}\right] \mathcal{B}^{T} \mathcal{W}_{k}^{T}
\end{aligned}
$$

where $\mathcal{H}=\mathcal{X}^{-1} \mathcal{F}^{T}, \mathcal{U}$ satisfies the PSE

$$
\tilde{\mathcal{A}} \mathcal{U}-\sigma \mathcal{U} \sigma \mathcal{A}-\sigma \mathcal{H} \sigma \mathcal{F}=0
$$

and $\mathcal{V}_{k}, \mathcal{W}_{k}, k=0, \cdots, K-1$ satisfy the PSE's

$$
\begin{aligned}
\tilde{\mathcal{A}} \mathcal{V}_{k}-\sigma \mathcal{V}_{k} \sigma \mathcal{A}-\sigma \mathcal{R}_{k} & =0 \\
\tilde{\mathcal{A}} \mathcal{W}_{k}-\sigma \mathcal{W}_{k} \sigma \mathcal{A}-\sigma \mathcal{Q}_{k} & =0
\end{aligned}
$$

with $\mathcal{R}_{k}=\mathcal{I}_{k} \mathcal{X}^{T}$ and $\mathcal{Q}_{k}=-\mathcal{I}_{k} \mathcal{X}^{-1} \mathcal{X}^{-T} \mathcal{X}^{-1}$.

Proof: See Appendix I.

To compute for a given $\mathcal{G}$ the function $J$ and its gradient, we have to solve apparently $2(K+1)$ PSE's of essentially the same form as (6). By exploiting the linearity of the PSE, it is easy to observe that we can solve directly for

$$
\mathcal{Z}=\sum_{k=0}^{K-1}\left(\operatorname{tr}\left[X_{k}^{-T} X_{k}^{-1}\right] \mathcal{V}_{k}+\operatorname{tr}\left[X_{k}^{T} X_{k}\right] \mathcal{W}_{k}\right)
$$

which satisfies the PSE

$$
\tilde{\mathcal{A}} \mathcal{Z}-\sigma \mathcal{Z} \sigma \mathcal{A}-\sigma \mathcal{P}=0
$$

where

$$
\mathcal{P}=\sum_{k=0}^{K-1}\left(\operatorname{tr}\left[X_{k}^{-T} X_{k}^{-1}\right] \mathcal{R}_{k}+\operatorname{tr}\left[X_{k}^{T} X_{k}\right] \mathcal{Q}_{k}\right)
$$

The corresponding expression of the gradient is

$$
\nabla_{\mathcal{G}} J=(1-\alpha)\left(\mathcal{H}^{T}-\mathcal{B}^{T} \mathcal{U}^{T}\right)+\alpha \mathcal{B}^{T} \mathcal{Z}^{T}
$$

Further, we can even solve instead of (8) and (11) a single PSE of the form

$$
\tilde{\mathcal{A}} \tilde{\mathcal{U}}-\sigma \tilde{\mathcal{U}} \sigma \mathcal{A}-\sigma \mathcal{S}=0
$$


where $\mathcal{S}=-(1-\alpha) \mathcal{H} \mathcal{F}+\alpha \mathcal{P}$, with $\mathcal{P}$ given by (12). The expression of the gradient is now

$$
\nabla_{\mathcal{G}} J=(1-\alpha) \mathcal{H}^{T}+\mathcal{B}^{T} \tilde{\mathcal{U}}^{T}
$$

A somewhat simpler expression for the free term of the PSE (14) results by employing an alternative cost function $J^{\prime}$, which essentially leads to the same results. The proposed modified cost function is

$$
\begin{aligned}
J^{\prime}= & \alpha \frac{1}{2} \sum_{k=0}^{K-1}\left(\operatorname{tr}\left[X_{k}^{T} X_{k}\right]+\operatorname{tr}\left[X_{k}^{-T} X_{k}^{-1}\right]\right) \\
& +(1-\alpha) \frac{1}{2} \sum_{k=0}^{K-1} \operatorname{tr}\left[F_{k}^{T} F_{k}\right]
\end{aligned}
$$

and can be expressed by using the script notation as

$$
\begin{aligned}
J^{\prime}= & \alpha\left(\frac{1}{2} \operatorname{tr}\left[\mathcal{X}^{T} \mathcal{X}\right]+\frac{1}{2} \operatorname{tr}\left[\mathcal{X}^{-T} \mathcal{X}^{-1}\right]\right) \\
& +(1-\alpha) \frac{1}{2} \operatorname{tr}\left[\mathcal{F}^{T} \mathcal{F}\right] .
\end{aligned}
$$

We have the following result for the expression of the gradient.

Proposition 2: Let $\mathcal{F}$ be the periodic feedback computed as in (5), assigning the desired characteristic values $\Gamma$ for given $\tilde{\mathcal{A}}$ and $\mathcal{G}$. Then, the gradient of $J^{\prime}$ with respect to $\mathcal{G}$ is given by

$$
\nabla_{\mathcal{G}} J^{\prime}=(1-\alpha) \mathcal{H}^{T}+\mathcal{B}^{T} \mathcal{U}^{T}
$$

where $\mathcal{H}=\mathcal{X}^{-1} \mathcal{F}^{T}$, and $\mathcal{U}$ satisfies the PSE (14) with

$$
\mathcal{S}=-(1-\alpha) \mathcal{H} \mathcal{F}+\alpha\left(\mathcal{X}^{T}-\mathcal{X}^{-1} \mathcal{X}^{-T} \mathcal{X}^{-1}\right)
$$

Proof: The proof is very similar to that of Proposition 1 and relies on the above-employed technique to linearly combine the solutions of several Sylvester equations differing only in their free terms.

Having explicit analytical expressions for the function and its gradient, it is easy to employ any gradient-based technique to minimize $J$ or $J^{\prime}$. Because the dimension of the minimization problem $K \mathrm{~nm}$ is potentially large, a particularly well-suited class of methods to solve our problem is the class of unconstrained descent methods, as, for instance, the limited memory BFGS method [8] used in conjunction with a line search procedure with guaranteed decrease, as that described in [12]. Both methods are implemented within the MINPACK-2 project (the successor of MINPACK-1 [11]) offering a convenient reverse communication interface that allows an easy implementation of function and gradient computations. The guaranteed decrease feature of these methods ensures that for $\alpha>0$ the condition numbers $\kappa_{F}\left(X_{k}\right)$ decrease and thus the solution $\mathcal{X}$ of (6) remains invertible at each iteration once an invertible solution has been determined at the first iteration.

\section{NUMERICAL ASPECTS}

In this section, we address the efficient calculation of the functions and gradients necessary in using gradient-based methods. For each function/gradient evaluation, the main computation is the solution of two PSE's, both essentially of the form

$$
\mathcal{A} \mathcal{X}-\sigma \mathcal{X} \tilde{\mathcal{A}}+\mathcal{S}=0
$$

To ensure a satisfactory performance, these computations must be done efficiently by using numerically reliable algorithms. The key observation, which allows a drastic reduction of the computational cost, is that, analogously to the standard case [15], both cost functions $J$ and $J^{\prime}$ are invariant to an orthogonal similarity transformation. Thus, if $\mathcal{F}$ is the optimal feedback for the original pair $(\mathcal{A}, \mathcal{B})$, then $\hat{\mathcal{F}}=\mathcal{F} \mathcal{Z}^{T}$ is the optimal feedback for the transformed pair $(\hat{\mathcal{A}}, \hat{\mathcal{B}})=\left(\sigma \mathcal{Z}^{T} \mathcal{A} \mathcal{Z}, \sigma \mathcal{Z}^{T} \mathcal{B}\right)$, where $\mathcal{Z}$ is an orthogonal matrix. By using such a transformation, we can tremendously reduce the computational effort necessary to repeatedly solve PSE's with the same coefficient matrices.

Recall that, by assumption, $\tilde{\mathcal{A}}$ in (19) is in a real Jordan form, having only $1 \times 1$ or $2 \times 2$ possibly nonzero diagonal blocks. To simplify further the solution of (19), we can determine an orthogonal $\mathcal{Z}$ such that $\hat{\mathcal{A}}=\sigma \mathcal{Z}^{T} \mathcal{A} \mathcal{Z}$ is in a periodic Schur form (PSF) [2], where $\hat{A}_{0}$ is in a real Schur form and the matrices $\hat{A}_{k}$ for $k=1, \cdots, K-1$ are upper triangular. By multiplying (19) with $\sigma \mathcal{Z}^{T}$ from left, one obtains a reduced PSE

$$
\hat{\mathcal{A}} \hat{\mathcal{X}}-\sigma \hat{\mathcal{X}} \tilde{\mathcal{A}}+\hat{\mathcal{S}}=0
$$

where $\hat{\mathcal{X}}=\mathcal{Z}^{T} \mathcal{X}$ and $\hat{\mathcal{S}}=\sigma \mathcal{Z}^{T} \mathcal{S}$. Notice that by this transformation the resulted transformed PSE (20) has exactly the same form as the original one in (19). After solving (20) for $\hat{\mathcal{X}}$, the solution of (19) results as $\mathcal{X}=\mathcal{Z} \hat{\mathcal{X}}$.

For the solution of the reduced PSE (20), an efficient method can be devised along the lines of the more general approach proposed in [3]. This procedure is described in Appendix II (see also [16] for additional details). The procedure in Appendix II can be further specialized to exploit the block-diagonal Jordan structure of $\tilde{\mathcal{A}}$. In this case, it performs about $0.5 K n^{3}$ floating-point operations (flops) to solve one reduced PSE of the form (20). By assuming $\mathcal{A}$ already reduced to the PSF, the total cost to evaluate either $J$ or $J^{\prime}$ and their gradients is about $4 K n^{3}$ flops, from which about $3 K n^{3}$ flops are necessary to form the free term of (20). Note that, without the preliminary reduction to the PSF, each function and gradient evaluation would require at least $12 \mathrm{Kn}^{3}$ additional flops.

The preliminary orthogonal transformation is also useful to remove the restrictive condition $\Lambda\left(\Phi_{A}(K, 0)\right) \cap \Lambda\left(\Phi_{\tilde{A}}(K, 0)\right)=\emptyset$ and thus to allow a partial eigenvalue assignment. By using a periodic orthogonal similarity transformation

$$
Z_{k+1}^{T} A_{k} Z_{k}=\left[\begin{array}{cc}
A_{k, 11} & A_{k, 12} \\
0 & A_{k, 22}
\end{array}\right], \quad Z_{k+1}^{T} B_{k}=\left[\begin{array}{c}
B_{k, 1} \\
B_{k, 2}
\end{array}\right]
$$

we can reduce the matrix $\mathcal{A}$ to an ordered PSF (see [2]), where the characteristic values of $\mathcal{A}_{11}$ are those corresponding to satisfactory poles (which will be kept unmodified), while the characteristic values of $\mathcal{A}_{22}$ will be moved to desired values. Now, we can compute by using gradient search techniques the solution $\mathcal{F}_{2}$ of a reduced-order PEAP such that the characteristic values of $\mathcal{A}_{22}+\mathcal{B}_{2} \mathcal{F}_{2}$ are assigned to desired locations. The final $K$-periodic state feedback results as $F_{k}=\left[\begin{array}{ll}0 & F_{k, 2}\end{array}\right] Z_{k}^{T}$.

\section{NUMERICAL EXAMPLES}

Example 1: This is a constant system example from [9] with

$$
A_{k}=\left[\begin{array}{ccc}
e & 0 & 0 \\
0 & e^{-1} & 0 \\
0 & 0 & 1
\end{array}\right], \quad B_{k}=\left[\begin{array}{cc}
e-1 & 0 \\
1 & 1-e^{-1} \\
1 & 0
\end{array}\right]
$$

for $k=0,1$. We assigned the poles at $\Gamma=\{0.6,0.7,-0.7\}$, and we computed for $\alpha=0,0.5$, and 1 the optimum periodic feedback by minimizing both $J$ and $J^{\prime}$. For each solution, we computed the resulting feedback norm $\|\mathcal{F}\|_{F}$ as well as the two-norm and Frobenius-norm condition numbers $\kappa_{2}\left(V_{k}\right)$ and $\kappa_{F}\left(V_{k}\right)$, where $V_{k}$ is the eigenvector 
TABLE I

RESULTS FOR EXAMPLE 1

\begin{tabular}{lccccc}
\hline \multicolumn{1}{c}{ Criteria } & $\|\mathcal{F}\|_{\mathcal{F}}$ & ${ }_{\kappa_{2}}\left(V_{0}\right)$ & ${ }_{\kappa_{2}}\left(V_{1}\right)$ & ${ }_{{ }_{F}}\left(V_{0}\right)$ & ${ }_{\kappa_{F}}\left(V_{1}\right)$ \\
\hline Ex. in $[9]$ & 4.83 & 9.12 & 4.33 & 20.18 & 9.12 \\
$J(\alpha=0)$ & 1.57 & 371.7 & 492.1 & 485.8 & 576.1 \\
$J(\alpha=0.5)$ & 4.45 & 8.0 & 6.30 & 15.40 & 11.09 \\
$J^{\prime}(\alpha=0.5)$ & 4.36 & 7.99 & 7.19 & 15.02 & 12.23 \\
$J(\alpha=1)$ & 5.13 & 8.06 & 6.23 & 15.44 & 11.01 \\
$J^{\prime}(\alpha=1)$ & 4.58 & 8.09 & 6.22 & 15.45 & 11.00 \\
$J^{\prime \prime}$ & 4.97 & 7.86 & 3.86 & 18.35 & 8.17 \\
\hline
\end{tabular}

matrix of the monodromy matrix $\Phi_{(A+B F)}(k+2, k)$. We also computed the optimal robust feedback minimizing $J^{\prime \prime}:=\Sigma \kappa_{2}\left(V_{k}\right)$. The results are summarized in Table I, where, for completeness, we also included results for the solution computed in [9].

It can be seen that, for this example, that the proposed approach computes better results in terms of $\sum \kappa_{2}\left(V_{k}\right)$ than the method of [9] for all considered nonzero values of $\alpha$. Moreover, in the case of minimizing $J^{\prime \prime}$, we even succeeded to achieve $\kappa_{2}\left(V_{0}\right)=\kappa_{2}\left(V_{1}\right)=1$, that is, to obtain orthogonal eigenvector matrices. The explanation for this lies in the parametric freedom of the problem. The PEAP can freely manipulate $N=n(K m-1)$ parameters of the total of $n m K$ parameters contained in $G_{k}$. The orthogonality of eigenvectors imposes additionally $\tilde{N}=K n(n-1) / 2$ algebraic equations. In our case, $N=9>\tilde{N}=6$; thus, orthogonal eigenvectors can be achieved by using our parametrization. For reference purposes, we give the values of the periodic feedback minimizing $J^{\prime \prime}$ :

$$
\begin{aligned}
& F_{1}=\left[\begin{array}{lll}
-2.9255 & 0.0979 & 0.4220 \\
-3.9647 & 0.5417 & 0.6962
\end{array}\right] \\
& F_{2}=\left[\begin{array}{lll}
-2.1550 & 0.1820 & 0.4041 \\
-4.1761 & 1.6204 & 1.3413
\end{array}\right] .
\end{aligned}
$$

Example 2: This is the multirate sampled-data system example of [9] with

$$
\begin{aligned}
A_{0}= & {\left[\begin{array}{lllll}
0 & 0 & 0 & 0 & 0 \\
0 & 0 & 0 & 0 & 0 \\
0 & 0 & e & 0 & 0 \\
0 & 0 & 0 & e^{-1} & 0 \\
0 & 0 & 0 & 0 & 1
\end{array}\right], \quad B_{0}=\left[\begin{array}{cc}
1 & 0 \\
0 & 1 \\
e-1 & 0 \\
0 & 1-e^{-1} \\
1 & 0
\end{array}\right] } \\
A_{1} & =\left[\begin{array}{llllll}
0 & 0 & 0 & 0 & 0 \\
0 & 1 & 0 & 0 & 0 \\
0 & 0 & e & 0 & 0 \\
0 & 1-e^{-1} & 0 & e^{-1} & 0 \\
0 & 0 & 0 & 0 & 1
\end{array}\right], \quad B_{1}=\left[\begin{array}{cc}
1 & 0 \\
0 & 0 \\
e-1 & 0 \\
0 & 0 \\
1 & 0
\end{array}\right] .
\end{aligned}
$$

We assigned the poles at $\Gamma=\{0.5,0.6,-0.6,0.7,-0.7\}$ obtaining results shown in Table II.

As can be observed from the table, all solutions computed for the nonzero values of $\alpha$ are qualitatively the same with the results of [9]. For reference purpose, we give the computed periodic feedback minimizing $J^{\prime \prime}$ :

$$
\begin{aligned}
& F_{1}=\left[\begin{array}{llllr}
0.2687 & -0.0243 & -2.9971 & 0.0256 & 0.3901 \\
0.0450 & -2.2733 & -0.1224 & 2.3404 & 0.0314
\end{array}\right] \\
& F_{2}=\left[\begin{array}{llllr}
-0.1446 & 0.0127 & -1.7855 & -0.0045 & 0.0725 \\
-0.1762 & 0.8689 & -0.4829 & -0.4798 & -0.6458
\end{array}\right] .
\end{aligned}
$$

TABLE II

RESULTS FOR EXAMPLE 2

\begin{tabular}{lccccc}
\hline \multicolumn{1}{c}{ Criteria } & $\|\mathcal{F}\|_{\mathcal{F}}$ & $\kappa_{2}\left(V_{0}\right)$ & ${ }_{\kappa_{2}}\left(V_{1}\right)$ & ${ }_{\kappa_{F}}\left(V_{0}\right)$ & ${ }_{\kappa_{F}}\left(V_{1}\right)$ \\
\hline Ex. in [9] & 4.59 & 1.67 & 11.17 & 3.27 & 12.26 \\
$J(\alpha=0)$ & 1.62 & 57.59 & 40.72 & 59.14 & 44.46 \\
$J(\alpha=0.5)$ & 2.84 & 2.89 & 2.77 & 4.32 & 4.21 \\
$J^{\prime}(\alpha=0.5)$ & 2.55 & 3.54 & 3.22 & 5.00 & 4.65 \\
$J(\alpha=1)$ & 3.60 & 2.87 & 2.89 & 4.24 & 4.24 \\
$J^{\prime}(\alpha=1)$ & 3.92 & 2.87 & 2.16 & 4.24 & 3.67 \\
$J^{\prime \prime}$ & 7.20 & 1.00 & 1.00 & 3.00 & 3.00 \\
\hline
\end{tabular}

\section{CONCLUSIONS}

We focused on developing a numerical approach to exploit the intrinsic nonuniqueness of the PEAP. One possibility to address the nonuniqueness is by formulating the PEAP as a minimum norm, robust pole assignment problem. By using a convenient parametrization, a solution of the PEAP is sought that minimizes a special cost function defined as a weighted sum between the Frobenius-norm of the periodic state feedback matrix and the condition numbers of the closed-loop eigenvector matrices. The derived explicit expressions for the cost function gradient allow the use of powerful gradient-search-based minimization techniques. The efficient evaluation of the cost function and its gradient is of paramount importance for the usefulness of the proposed approach. The main numerical aspects of these computations have been thoroughly discussed, including the efficient solution of the involved periodic Sylvester-equations. The presented numerical examples indicate that our method provides comparable or even better results than the recently proposed robust pole assignment method in [9]. The extension of the proposed approach to periodic systems with variable dimensions of the state and input vectors is apparently possible. This aspect is, however, still under investigation.

\section{APPENDIX I \\ PROOFS}

To prove Proposition 1, we need the following result.

Lemma 1: Let $A_{k}, B_{k}, C_{k}, D_{k}$ be $n \times n K$-periodic matrices, and let $\mathcal{X}$ be the solution of the PSE

$$
\mathcal{A X}+\sigma \mathcal{X} \mathcal{B}=\mathcal{C}
$$

Then

$$
\operatorname{tr}[\mathcal{D} \mathcal{X}]=\operatorname{tr}[\mathcal{U C}]
$$

where $\mathcal{U}$ satisfies the PSE

$$
\mathcal{B U}+\sigma \mathcal{U} \sigma \mathcal{A}=\sigma \mathcal{D} .
$$

Proof: To simplify the notation, take $\mathcal{Q}=\mathcal{D}^{T}$. For an arbitrary $K$-periodic matrix $Y_{k}$, we shall also use the notation $\overline{\bar{y}}$ to denote

$$
\overline{\bar{y}}=\left[\begin{array}{c}
\operatorname{vec}\left(Y_{0}\right) \\
\vdots \\
\operatorname{vec}\left(Y_{K-1}\right)
\end{array}\right]
$$

where the operator $\operatorname{vec}(\cdot)$ generates a vector from the stacked columns of a matrix. By using this notation, we can express the solution of the PSE (21) as

$$
\overline{\bar{x}}=P^{-1} \overline{\bar{c}}
$$


where, for $K=3$, say, $P$ has the form

$$
P=\left[\begin{array}{ccc}
I \otimes A_{1} & B_{1}^{T} \otimes I & 0 \\
0 & I \otimes A_{2} & B_{2}^{T} \otimes I \\
B_{3}^{T} \otimes I & 0 & I \otimes A_{3}
\end{array}\right]
$$

With the above expression for $\overline{\bar{x}}$, we obtain successively

$$
\operatorname{tr}[\mathcal{D} \mathcal{X}]=\operatorname{tr}\left[\mathcal{Q}^{T} \mathcal{X}\right]=\overline{\bar{q}}^{T} \overline{\bar{x}}=\overline{\bar{q}}^{T} P^{-1} \overline{\bar{c}}=\overline{\bar{y}}^{T} \overline{\bar{c}}
$$

where $\overline{\bar{y}}$ satisfies $P^{T} \overline{\bar{y}}=\overline{\bar{q}}$. Looking at

$$
P^{T}=\left[\begin{array}{ccc}
I \otimes A_{1}^{T} & 0 & B_{3} \otimes I \\
B_{1} \otimes I & I \otimes A_{2}^{T} & 0 \\
0 & B_{2} \otimes I & I \otimes A_{3}^{T}
\end{array}\right]
$$

we observe that $P^{T} \overline{\bar{y}}=\overline{\bar{q}}$ corresponds to the PSE

$$
\mathcal{Y} \mathcal{B}^{T}+\sigma \mathcal{A}^{T} \sigma \mathcal{Y}=\sigma \mathcal{Q}
$$

or, after transposing

$$
\mathcal{B Y}^{T}+\sigma \mathcal{Y}^{T} \sigma \mathcal{A}=\sigma \mathcal{Q}^{T}
$$

This equation is precisely the PSE (22), with the obvious replacements $\mathcal{Y}^{T}=\mathcal{U}$ and $\mathcal{Q}^{T}=\mathcal{D}$. We further have

$$
\operatorname{tr}[\mathcal{D} \mathcal{X}]=\overline{\bar{y}}^{T} \overline{\bar{c}}=\operatorname{tr}\left[\mathcal{Y}^{T} \mathcal{C}\right]=\operatorname{tr}[\mathcal{U C}]
$$

Proof of Proposition 1: We can write the cost function (4) in the form

$$
J=(1-\alpha) J_{1}+2 \alpha \sum_{k=0}^{K-1} J_{2}^{(k)} J_{3}^{(k)}
$$

where $J_{1}=(1 / 2) \operatorname{tr}\left[\mathcal{F}^{T} \mathcal{F}\right], J_{2}^{(k)}=(1 / 2) \operatorname{tr}\left[\mathcal{I}_{k} \mathcal{X}^{T} \mathcal{X}\right]$, and $J_{3}^{(k)}=$ $(1 / 2) \operatorname{tr}\left[\mathcal{I}_{k} \mathcal{X}^{-T} \mathcal{X}^{-1}\right]$. It follows that

$$
\nabla_{\mathcal{G}} J=(1-\alpha) \nabla_{\mathcal{G}} J_{1}+2 \alpha \sum_{k=0}^{K-1}\left(\nabla_{\mathcal{G}} J_{2}^{(k)} J_{3}^{(k)}+J_{2}^{(k)} \nabla_{\mathcal{G}} J_{3}^{(k)}\right)
$$

For $J_{1}=(1 / 2) \operatorname{tr}\left[\mathcal{F}^{T} \mathcal{F}\right]$, we deduce the gradient $\nabla_{\mathcal{G}} J_{1}$ from the first-order variation

$$
\Delta J_{1}:=\operatorname{tr}\left[\left(\nabla_{\mathcal{G}} J_{1}\right)^{T} \Delta \mathcal{G}\right]=\operatorname{tr}\left[\mathcal{F}^{T} \Delta \mathcal{F}\right]
$$

From (5), we get

$$
\Delta \mathcal{F}=\Delta \mathcal{G} \mathcal{X}^{-1}-\mathcal{G} \mathcal{X}^{-1} \Delta \mathcal{X} \mathcal{X}^{-1}
$$

and we have successively

$$
\begin{aligned}
\Delta J_{1} & =\operatorname{tr}\left[\mathcal{F}^{T}(\Delta \mathcal{G}-\mathcal{F} \Delta \mathcal{X}) \mathcal{X}^{-1}\right] \\
& =\operatorname{tr}\left[\mathcal{X}^{-1} \mathcal{F}^{T}(\Delta \mathcal{G}-\mathcal{F} \Delta \mathcal{X})\right] \\
& \left.=\operatorname{tr}\left[\mathcal{X}^{-1} \mathcal{F}^{T} \Delta \mathcal{G}\right]-\operatorname{tr}\left[\mathcal{X}^{-1} \mathcal{F}^{T} \mathcal{F} \Delta \mathcal{X}\right)\right] .
\end{aligned}
$$

From (6), it follows that $\Delta \mathcal{X}$ satisfies the PSE

$$
\mathcal{A} \Delta \mathcal{X}-\sigma \Delta \mathcal{X} \tilde{\mathcal{A}}+\mathcal{B} \Delta \mathcal{G}=0 .
$$

By using Lemma 1, we can write

$$
\operatorname{tr}\left[\mathcal{X}^{-1} \mathcal{F}^{T} \mathcal{F} \Delta \mathcal{X}\right]=\operatorname{tr}[\mathcal{U B} \Delta \mathcal{G}]
$$

where $\mathcal{U}$ satisfies the PSE

$$
\tilde{\mathcal{A}} \mathcal{U}-\sigma \mathcal{U} \sigma \mathcal{A}-\sigma\left(\mathcal{X}^{-1} \mathcal{F}^{T} \mathcal{F}\right)=0
$$

which, with $\mathcal{H}=\mathcal{X}^{-1} \mathcal{F}^{T}$, is in fact (8). We further obtain

$$
\Delta J_{1}=\operatorname{tr}[(\mathcal{H}-\mathcal{U B}) \Delta \mathcal{G}]
$$

from which the gradient of $J_{1}$ results as

$$
\nabla_{\mathcal{G}} J_{1}=\mathcal{H}^{T}-\mathcal{B}^{T} \mathcal{U}^{T}
$$

We compute next the gradient of $J_{2}^{(k)}=(1 / 2) \operatorname{tr}\left[\mathcal{I}_{k} \mathcal{X}^{T} \mathcal{X}\right]$. As before, by using Lemma 1 , we have

$$
\left.\Delta J_{2}^{(k)}=\operatorname{tr}\left[\mathcal{I}_{k} \mathcal{X}^{T} \Delta \mathcal{X}\right)\right]=\operatorname{tr}\left[\mathcal{V}_{k} \mathcal{B} \Delta \mathcal{G}\right]
$$

where $\mathcal{V}_{k}$ satisfies the PSE (9) with $\mathcal{R}_{k}=\mathcal{I}_{k} \mathcal{X}^{T}$. From $\Delta J_{2}^{(k)}:=$ $\operatorname{tr}\left[\left(\nabla_{\mathcal{G}} J_{2}^{(k)}\right)^{T} \Delta \mathcal{G}\right]$, we obtain the gradient as

$$
\nabla_{\mathcal{G}} J_{2}^{(k)}=\mathcal{B}^{T} \mathcal{V}_{k}^{T}
$$

For the term $J_{3}^{(k)}=(1 / 2) \operatorname{tr}\left[X_{k}^{-T} X_{k}^{-1}\right]$, we use again Lemma 1 and we have successively

$$
\begin{aligned}
\Delta J_{3}^{(k)} & =\operatorname{tr}\left[X_{k}^{-T} \Delta\left(X_{k}^{-1}\right)\right] \\
& =-\operatorname{tr}\left[X_{k}^{-T} X_{k}^{-1} \Delta X_{k} X_{k}^{-1}\right] \\
& =-\operatorname{tr}\left[X_{k}^{-1} X_{k}^{-T} X_{k}^{-1} \Delta X_{k}\right] \\
& =-\operatorname{tr}\left[\mathcal{I}_{k} \mathcal{X}^{-1} \mathcal{X}^{-T} \mathcal{X}^{-1} \Delta \mathcal{X}\right] \\
& =\operatorname{tr}\left[\mathcal{W}_{k} \mathcal{B} \Delta \mathcal{G}\right]
\end{aligned}
$$

where $\mathcal{W}_{k}$ satisfies (10) with $\mathcal{Q}_{k}=-\mathcal{I}_{k} \mathcal{X}^{-1} \mathcal{X}^{-T} \mathcal{X}^{-1}$. We obtain the gradient of $J_{3}^{(k)}$ as

$$
\nabla_{\mathcal{G}} J_{3}^{(k)}=\mathcal{B}^{T} \mathcal{W}_{k}^{T}
$$

To obtain (7), we assemble $\nabla_{\mathcal{G}} J$ according to (23) by using (24)-(26).

\section{APPENDIX II SOLUTION OF PSE'S}

Let $A_{k} \in \mathbb{R}^{n \times n}, B_{k} \in \mathbb{R}^{m \times m}$, and $C_{k} \in \mathbb{R}^{n \times m}$ be $K$-periodic matrices. In this appendix, we consider the solution of PSE of the form

$$
\mathcal{A X}+\sigma \mathcal{X} \mathcal{B}=\mathcal{C}
$$


where $\mathcal{X}$ is the periodic solution. The solution method presented here is a direct generalization of the well-known Bartels-Stewart method [1] and has been discussed in a more general setting in [3]. The method presented here can be seen as specialization of the procedure of [3] for the case in which both $\mathcal{A}$ and $\mathcal{B}$ are reduced to PSF. Assume $\mathcal{A}$ and $\mathcal{B}$ are already in PSF and partitioned according to their PSF

$$
\begin{aligned}
A_{k} & =\left[\begin{array}{cccc}
A_{k, 11} & A_{k, 12} & \cdots & A_{k, 1 \bar{n}} \\
0 & A_{k, 22} & \cdots & A_{k, 2 \bar{n}} \\
\vdots & \vdots & \ddots & \vdots \\
0 & 0 & \cdots & A_{k, \bar{n}}
\end{array}\right] \\
B_{k} & =\left[\begin{array}{cccc}
B_{k, 11} & B_{k, 12} & \cdots & B_{k, 1 \bar{m}} \\
0 & B_{k, 22} & \cdots & B_{k, 2 \bar{m}} \\
\vdots & \vdots & \ddots & \vdots \\
0 & 0 & \cdots & B_{k, \bar{m}}
\end{array}\right] .
\end{aligned}
$$

Let us partition analogously the matrix $X_{k}$

$$
X_{k}=\left[\begin{array}{cccc}
X_{k, 11} & X_{k, 12} & \cdots & X_{k, 1 \bar{m}} \\
X_{k, 21} & X_{k, 22} & \cdots & X_{k, 2 \bar{m}} \\
\vdots & \vdots & \ddots & \vdots \\
X_{k, \bar{n} 1} & X_{k, \bar{n} 2} & \cdots & X_{k, \bar{n} \bar{m}}
\end{array}\right] .
$$

From (27), it follows that the $(r, l)$ th block $\mathcal{X}_{r l}$ satisfies the PSE

$$
\mathcal{A}_{r r} \mathcal{X}_{r l}+\sigma \mathcal{X}_{r l} \mathcal{B}_{l l}=\mathcal{M}_{r l}
$$

where

$$
\mathcal{M}_{r l}=\mathcal{C}_{r l}-\sum_{i=r+1}^{\bar{n}} \mathcal{A}_{r i} \mathcal{X}_{i l}-\sum_{j=1}^{l-1} \sigma \mathcal{X}_{r j} \mathcal{B}_{j l}
$$

The above equations can be solved successively for $\mathcal{X}_{\bar{n} 1}, \cdots, \mathcal{X}_{21}$, $\mathcal{X}_{11}, \mathcal{X}_{\bar{n} 2}, \cdots, \mathcal{X}_{12}, \cdots, \mathcal{X}_{\bar{n}}, \cdots, \mathcal{X}_{1 \bar{m}}$, and this leads to the following procedure.

Algorithm: Periodic Schur method to solve PSE.

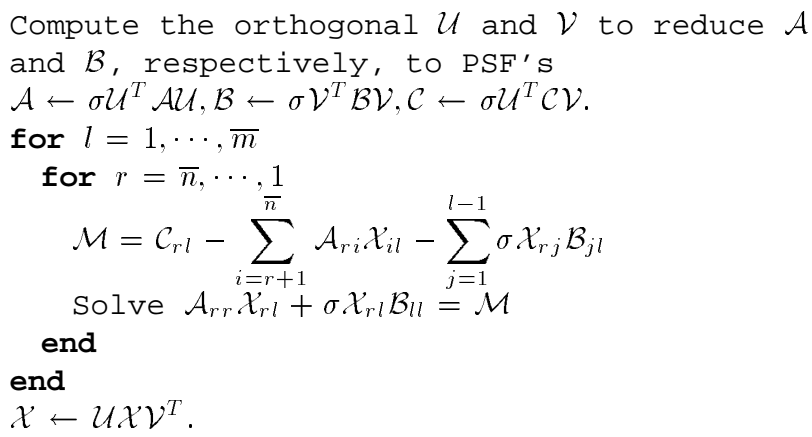

This algorithm allows us to overwrite $\mathcal{C}$ with the computed solution $\mathcal{X}$. Thus, the additional storage necessary to implement this algorithm is $K\left(n^{2}+m^{2}\right)$ locations. If we neglect the effort to solve the low-order PSE's, then the core algorithm performs about $0.5 K\left(m^{2} n+m n^{2}\right)$ flops. The total number of operations to solve a PSE is about $K\left(10 n^{3}+\right.$ $\left.10 m^{3}+2.5 n^{2} m+2.5 \mathrm{~nm}^{2}\right)$ flops.

The computation of the solution $\mathcal{X}_{r l}$ of (28) requires the efficient solution of low-order PSE's of the form

$$
E_{k} Y_{k}+Y_{k+1} F_{k}=G_{k}, \quad k=0, \cdots, K-1 ; Y_{0}=Y_{K}
$$

where $E_{k} \in \mathrm{R}^{n_{1} \times n_{1}}, F_{k} \in \mathrm{R}^{n_{2} \times n_{2}}$, and $G_{k} \in \mathrm{R}^{n_{1} \times n_{2}}$ with $1 \leq$ $n_{1}, n_{2} \leq 2$. An efficient method to solve such equations is discussed in [16]. The method relies essentially on rewriting the above equations with the help of Kronecker products as a system of $n_{1} n_{2} K$ simultaneous linear equations $H y=g$, where the coefficient matrix $H$ is a structured sparse matrix. Ignoring the sparse structure of $H$ in solving $H y=g$ leads, even for moderate values of $K$, to rather expensive computations. To exploit the structure of $H$, we can arrange, by an appropriate grouping of unknowns in the vector $y$ and by a suitable ordering of the equations, to obtain the coefficient matrix $H$ in a block-Hessenberg form. Then, a specialized block variant of the Gaussian elimination algorithm is used to solve $H y=g$. For details, see [16].

\section{REFERENCES}

[1] R. H. Bartels and G. W. Stewart, "Algorithm 432: Solution of the matrix equation $\mathrm{AX}+\mathrm{XB}=\mathrm{C}$," Commun. ACM, vol. 15, pp. 820-826, 1972.

[2] A. W. Bojanczyk, G. Golub, and P. Van Dooren, "The periodic Schur decomposition. Algorithms and applications," in Proc. SPIE Conf., July 1992, pp. 31-42.

[3] R. Byers and N. Rhee, "Cyclic Schur and Hessenberg-Schur numerical methods for solving periodic Lyapunov and Sylvester equations," Tech. Rep., Dept. Math., Univ. Missouri, Kansas City, June 1995.

[4] O. M. Grasselli and S. Longhi, "Pole-placement for nonreachable periodic discrete-time systems," Math. Contr. Signals Syst., vol. 4, pp. 439-455, 1991

[5] J. Kautsky, N. K. Nichols, and P. Van Dooren, "Robust pole assignment in linear state feedback," Int. J. Contr., vol. 41, pp. 1129-1155, 1985.

[6] L. H. Keel, J. A. Fleming, and S. P. Bhattacharyya, "Minimum norm pole assignment via Sylvester's equation," in Linear Algebra and Its Role in Systems Theory, 1995, vol. 47, AMS Contemporary Mathematics, pp. 265-272.

[7] M. Kono, "Eigenvalue assignment in linear periodic discrete-time systems," Int. J. Contr., vol. 32, pp. 149-158, 1980.

[8] D. C. Liu and J. Nocedal, "On the limited memory BFGS method for large scale optimization," Mathematical Programming, vol. 45, pp. 503-528, 1989.

[9] S. Longhi and R. Zulli, "A note on robust pole assignment for periodic systems," IEEE Trans. Automat. Contr., vol. 41, pp. 1493-1497, 1996.

[10] V. L. Mehrmann and H. Xu, "An analysis of the pole placement problem. II. The multi input case," Electron. Trans. Numer. Anal., vol. 5, 1998. Available: http://etna.mcs.kent.edu/.

[11] J. J. Moré, "User's Guide for MINPACK-1," Argonne Nat. Lab., Argone, IL, Appl. Math. Division Rep. ANL-80-74, 1980.

[12] J. J. Moré and D. J. Thuente, "On line search algorithms with guaranteed sufficient decrease," ACM Trans. Math. Software, vol. 20, pp. 286-307, 1994

[13] R. V. Patel, A. J. Laub, and P. Van Dooren, Eds., Numerical Linear Algebra Techniques for Systems and Control. Piscataway, NJ: IEEE Press, 1994.

[14] J. Sreedhar and P. Van Dooren, "Pole placement via the periodic Schur decomposition," in Proc. 1993 Amer. Contr. Conf., San Francisco, CA, 1993, pp. 1563-1567.

[15] A. Varga, "Parametric methods for pole assignment," in Proc. Eur. Contr. Conf., Brussels, Belgium, 1997, pp. 1-6. CDRM Paper FR-M H2.

[16] _ - "Periodic Lyapunov equations: Some applications and new algorithms," Int. J. Contr., vol. 67, pp. 69-87, 1997. 